\title{
Numerical Simulation for Optimum Seeding Operation Using Liquid Carbon Dioxide
}

\author{
Misato NAGATA ${ }^{*}, K_{\text {Kenji WAKIMIZU }}^{*}$, Koji NISHIYAMA ${ }^{* *}$, Norihiko FUKUTA ${ }^{* * *}$ \\ and Taichi MAKI \\ "Faculty of Agriculture, Kyushu University, Fukuoka, Japan \\ "*Faculty of Engineering, Kyushu University, Fukuoka, Japan \\ ".*. Department of Meteorology, University of Utah, Salt Lake City, USA
}

\begin{abstract}
In order to effectively convert a large amount of cloud water into precipitation, LOLEPSHIN (Low Level Penetration Seeding of Homogeneous Ice Nucleant) using liquid carbon dioxide (LC) was suggested. In this paper, consideration of an optimum seeding rate for a successful seeding operation was done using a timedependent dynamic and microphysical thermal model. The results of the model indicated that an optimum seeding rate should be determined on the basis of consuming efficiency and ice particles properties as well as the detection of radar echo measuring the cloud thickness. Therefore, application of the simplified microphysical and dynamic formulation to a cloud seeding operation is required for providing useful information to judge if a seeding operation leads to a significant result.
\end{abstract}

Key words: Ice crystal, Liquid carbon dioxide (LC), Microphysics, Seeding

\section{Introduction}

In order to effectively convert large amount of cloud water into precipitation, it is important that artificially formed ice particles attains sufficient size to be detected by meteorological radar at the cloud top and large enough fall velocity to arrive at the ground within the period of a seeding operation.

To achieve that goal, Fukuta (1999) suggested LOLEPSHIN (Low Level Penetration Seeding of Homogeneous Ice Nucleant) using liquid carbon dioxide (LC), which causes strong evaporative cooling reaching -90 degrees and the subsequent generation of approximately $10^{13}$ ice particles per gram of LC by homogeneous nucleation. The method is designed to induce a large amount of precipitation through two basic methods consisting of RETHIT (Roll-up Expansion of Twin Horizontal Ice Crystal Thermal) and FILAS (Falling-growth Induced Lateral Air Spreading) as shown in Fukuta (1996).

This seeding method was applied to supercooled convective clouds under the outbreak of a cold air mass from the Siberia in Northern Kyushu, Japan, on February 2, 1999. Under that condition, tracing of radar images confirmed the formation of two artificial radar echoes. The echoes expanded horizontally and reached to approximately $17 \mathrm{~km}$ in the upper width and took a unique mushroom shape with the echo symmetrically spread on both sides of the upper portion of the artificially induced ice crystal thermal.
In this paper, consideration of an optimum seeding rate for successful seeding operation was done using a time-dependent dynamic and microphysical thermal model, comparing the results with the abovementioned seeding experiment.

\section{Detail of LOLEPTHIN}

\subsection{Detail of LOLEPSHIN}

LOLEPSHIN is designed to maximize the efficiency of seeding through an LC seeding operation by an aircraft at a low supercooled portion of less than 0 degrees in a young developing cumulus containing sufficient supercooled liquid water content. In the cumulus, artificially formed ice particles can spend a relatively long time at growing into sufficient size to fall out within the limited lifetime of the cumulus.

The first seeding reaction due to the injection of LC materials into supercooled clouds is characterized by strong evaporation cooling reaching $\mathbf{- 9 0}$ degrees and the subsequent generation of approximately $10^{13}$ ice particles per gram of LC materials by homogeneous nucleation as shown by Fukuta (1988). The first reaction generates buoyant force due to the latent heat release and resultant thermal. During the ascent of the thermal after the injection of $\mathrm{LC}$, the number of artificially formed ice particles stays approximately constant and, therefore, no competition process for limited supercooled liquid water among ice particles occurs in the thermal. Therefore, the conservation of ice particles in the

Accepted on December 14, 2004 


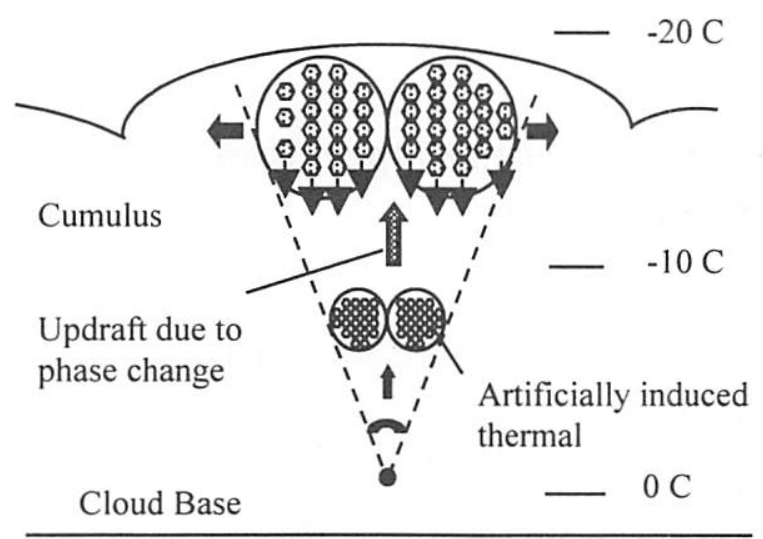

Fig. 1. RETHIT process

thermal provides an important advantage for effective growth of ice particles. From this point of view, the LC seeding method is more advantageous than the AgI seeding method, which causes an extreme competition process due to at drastic increase in the number of ice particles in the low temperature.

\subsection{RETHIT and FILAS processes}

The LOLEPSHIN method is designed to induce a large amount of precipitation through two basic processes. The first process is called RETHIT, which means Roll-up Expansion of Twin Horizontal Ice Crystal Thermal. The second process is called FILAS, which means Falling-growth Induced Lateral Air Spreading.

In the first process, RETHIT (see Fig. 1), ice particles form instantaneously by homogeneous nucleation after LC seeding and the resultant artificially induced thermal starts to go up with latent heat release due to the phase change of water vapor into ice. The thermal takes the shape of twodimensional twin cylinders perpendicular to the flight path and continues to go upward, expanding its volume at a constant vertical angle as shown by Scorer (1957) because of the continuous supply of buoyant energy generated by the latent heat release and the associated turbulent diffusion. The ice particles can grow into large enough size to fall out and to be detected by meteorological radar with little competition among ice particles until the thermal arrives at the cloud top because of no change in the number of artificially generated ice particles if a suitable seeding rate is selected.

In the FILAS process (see Fig. 2), after the thermal reaches a stable layer, the falling ice particles move horizontally toward both sides of the thermal due to lateral spreading. Then, the horizontally moving and falling ice particles cause an artificial secondary upward motion in the mature stage of the cumulus due to the balance between gravity resistance of ice particles and the latent heat release due to phase change of water vapor and the cloud volume continues to expand horizontally due to the existence of the stable layer around the cloud top until all of the available liquid water for the ice particles in the cumulus is converted into ice. In this stage, the ice

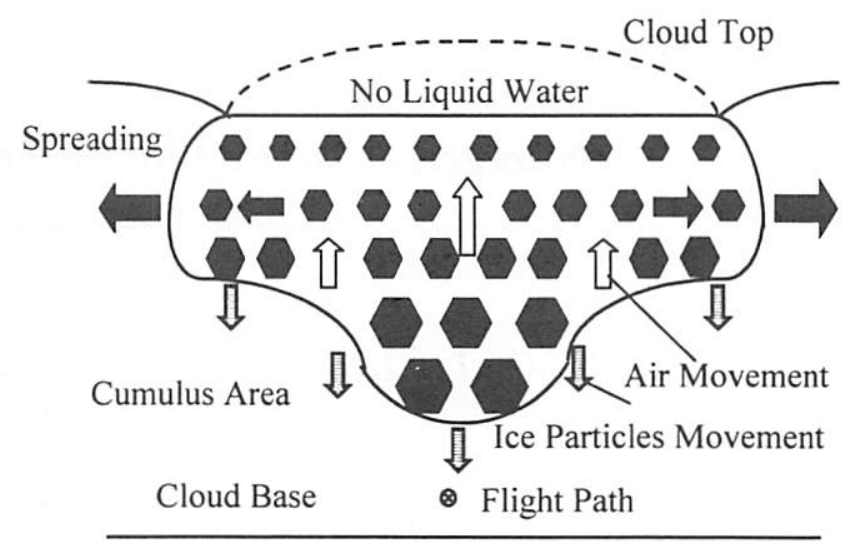

Fig. 2. FILAS process

particles continue to grow further by consuming additionally formed liquid cloud water by the secondary upward motion.

\section{Summary of LC Seeding Experiment}

It is expected that the outcome of the newly developed rainmaking operation by LOLEPSHIN (Fukuta, 1999) will contribute to a stable supply of springtime water resources in the northern Japan including Tokyo. In this study, based on the abovementioned explanation of snowfall induced by typical winter monsoons in Japan, a seeding operation in winter for springtime water resources will be discussed. A seeding operation in winter should select a developing cumulus in a cloud band induced by the winter monsoon. In the seeding operation, in particular, it should be determined that the selected target cumulus has sufficient cloud thickness and horizontal area in the developing stage for containing ample liquid water content.

A seeding experiment by LOLEPSHIN was carried out around Iki Island in northern Kyushu located in the west of Japan on February 2, 1999. The weather condition on this day was characterized by a typical winter monsoon. Therefore, there were many cloud bands induced by strong seasonal wind. In the experiment, developing supercooled cumuli approaching towards the north area of Kyushu Island were selected as targets. An operational aircraft penetrated horizontally into three selected clouds at a ground speed of $100 \mathrm{~m} / \mathrm{s}$ at -4 degrees $(1.2 \mathrm{~km}$ altitude) level and discharged LC at a rate of $6 \mathrm{~g} / \mathrm{s}$. Subsequently, GPS tracking of aircraft and Kyushu

University radar confirmed that the seeding operation succeeded in the generation of two artificial seeded echoes.

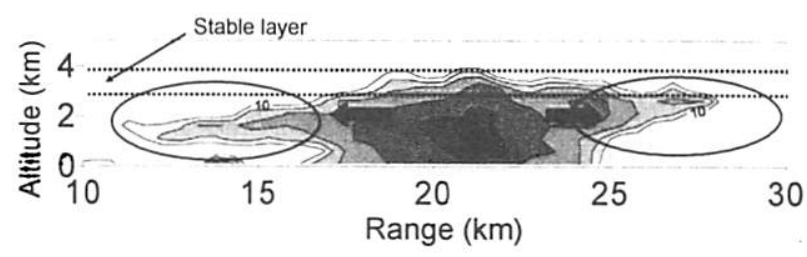

Fig. 3. Appearance of mushroom echo 


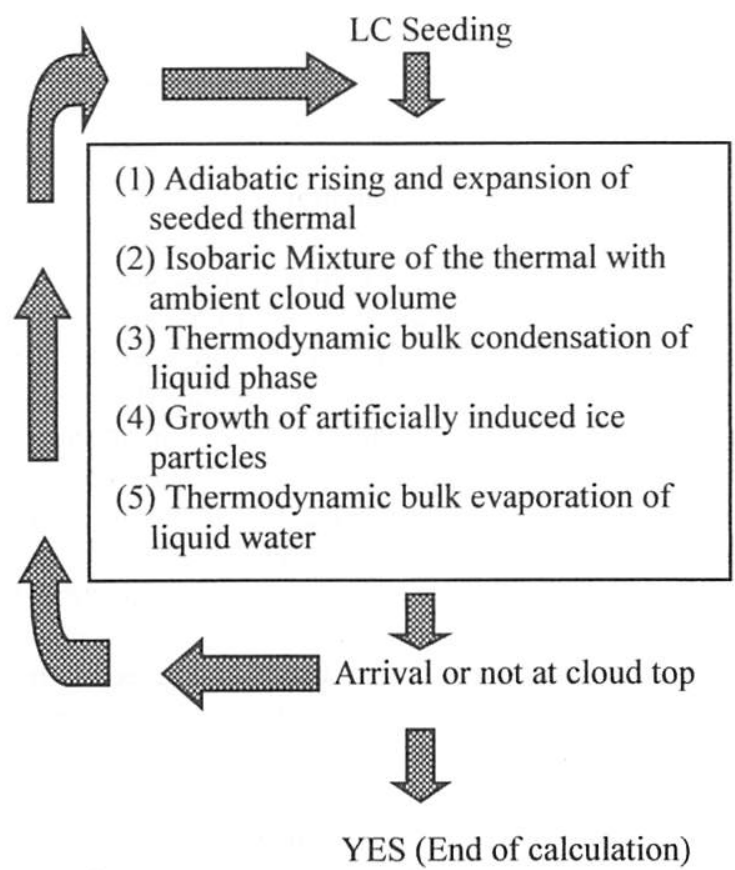

Fig. 4. Procedure for time-dependent numerical model calculation

In this seeding operation, one of the notable features was the appearance of a horizontally
Table 1. Liquid water content depend on cloud thickness

\begin{tabular}{|c|c|}
\hline Cloud thickness & Liquid water content \\
\hline $1.0 \mathrm{~km}$ & $0.65 \mathrm{~g} / \mathrm{m}^{3}$ \\
\hline $2.0 \mathrm{~km}$ & $1.13 \mathrm{~g} / \mathrm{m}^{3}$ \\
$3.0 \mathrm{~km}$ & $1.47 \mathrm{~g} / \mathrm{m}^{3}$ \\
$2.4 \mathrm{~km}$ & $1.28 \mathrm{~g} / \mathrm{m}^{3}$ \\
\hline
\end{tabular}

spreading echo like a mushroom (Fig. 3) up to approximately $15 \mathrm{~km}$ in the anvil and the subsequent horizontal expansion of the radar echo. The mushroom-type echo indicated falling and wide spreading of ice crystals grown into sufficient size to be detected by radar and, in other words, shows that a large amount of cloud volume can be converted into sufficient precipitation to bring significant water resources. Therefore, in order to achieve optimum seeding effects during RETHIT process it is necessary that artificially induced ice particles have sufficient mass and moderate number concentration to bring a radar echo when they reach the cloud top. In addition, it is necessary that ice particles grown in RETHIT have sufficient terminal velocity to reach

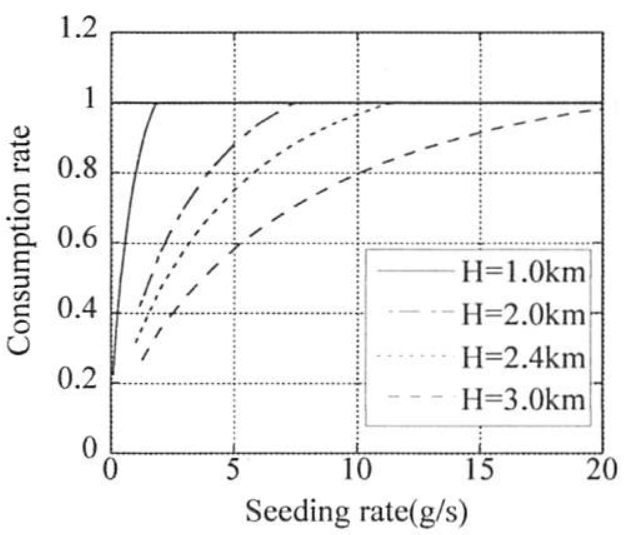

Fig. 6. Consumption rate of available ice water content

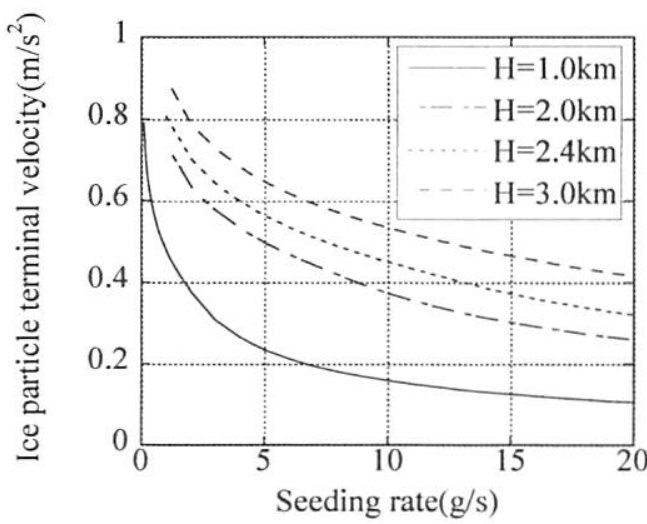

Fig. 8. The dependence of ice particles terminal velocity on seeding rate with cloud thickness

Fig. 7. The dependence of ice particles mass on seeding rate with cloud thickness 
the ground in the lifetime of a seeded cloud.

\section{Numerical Model}

\subsection{Summary of Numerical Model}

Measuring of the optimum seeding rate for a successful seeding operation was done using timedependent dynamic and microphysical thermal model according to Javanmard et al (1998).

The model incorporates five dynamic and microphysical procedures as shorn in Fig.4. The calculation continues until an artificial ice thermal arrives at a cloud top after seeding. In this study, the seeding level is set to be $1.2 \mathrm{~km}$ altitude $\left(-4^{\circ} \mathrm{C}\right)$. It is assumed that liquid water content is constant throughout the cloud volume and depends on the cloud thickness according to table.1.

Here, in order to check the consumption of liquid water content generated in the thermal by artificial ice particles, consumption rate is defined as follows;

$$
\gamma=\frac{W_{i}}{W_{i}+W_{l}}
$$

where $\gamma$ is consumption rate, $W_{i}$ is ice water content, $W_{L}$ is remaining liquid water content in the ice thermal.

\subsection{Results of calculation}

A requirement for an optimum seeding operation is the detection of an artificially induced radar echo from an observational point of view. An actual seeding operation with the seeding rate of $6 \mathrm{~g} / \mathrm{s}$ penetrating into a group of cumulus clouds with the thickness of approximately $2.4 \mathrm{~km}$ induced an echo intensity of 10 to $25 \mathrm{dBZ}$. The observed facts are roughly consistent with numerical results which indicate that the detection of an artificial radar echo requires seeding rates of less than $10 \mathrm{~g} / \mathrm{s}$ assuming the lowest limit of $10 \mathrm{dBZ}$ as shown in Fig. 5 .

However, it should be noted that the consuming efficiency of liquid water content among ice particles increases with an increase in seeding rate as shown by Fig. 6 and, on the contrary, mass and terminal velocity of ice particles decrease with an increase in the seeding rate as shown in Fig. 7 and 8. Therefore, the optimum seeding rate should be determined on the basis of consuming efficiency and ice particles properties as well as the detection by radar echo.

Next, dependence of seeding reaction on cloud thickness was investigated. A slight difference on numerical seeding results between 2 and $3 \mathrm{~km}$ thickness was recognized and, on the other hand, there was large difference between $1 \mathrm{~km}$ and more than $2 \mathrm{~km}$.

Therefore, a cloud thickness of more than $2 \mathrm{~km}$ is a requirement for a successful optimum seeding operation. On the other hand, a cumulus cloud with the thickness of $1 \mathrm{~km}$ has no optimum seeding condition because of lack of ice particle mass due to competitive growth for the limited liquid water content generated in a thin cumulus cloud.
From the above results, the numerical results of an LC seeding operation give some indications of an optimum seeding operation.

\section{Conclusion}

In order to effectively convert a large amount of cloud water into precipitation, LOLEPSHIN (Low Level Penetration Seeding of Homogeneous Ice Nucleant) using liquid carbon dioxide (LC) was suggested. In this paper, measuring of an optimum seeding rate for a successful seeding operation was done using a time-dependent dynamic and microphysical thermal model. The results of the model indicated that the optimum seeding rate should be determined on the basis of consuming efficiency and ice particles properties as well as the detection by radar echo considering the cloud thickness. Therefore, application of the simplified microphysical and dynamic formulation to a cloud seeding operation is required for providing useful information to judge if a seeding operation will lead to a significant result.

\section{References}

Fukuta, N., 1988: The Maximum rate of Homogeneous Ice Nucleant in Air by Cooling. Proceedings $12^{\text {th }}$ Intl. Conf. on Nucleation and Atmos Aerosols, Vienna, Springer-Verlag., pp.504-507.

Fukuta, N., 1996: Project Mountain Valley SunshineProgress in Science and Technology. J. Appl. Meteor, Vol.35, pp.1483-1493.

Fukuta, N., 1999: Feedbacked utilization of phase change energy for lifting, turbulent generation and spreading of seeding ice thermal and optimization of the seeding effect. Preprint, $7^{\text {th }}$ WMO Scientific Conf on Wea. Mod., Chiang Mai, pp.363-366.

Javanmard, S., Nishiyama, K., Fukuta, N., K, Wakimizu., Y, Suzuki 1998: Numerical modeling for roll-up expansion of twin horizontal ice crystal thermals in liquid carbon dioxide seeding. Preprints $14^{\text {th }}$ Conf on Planned \& Inadvertent Weather Modification, 17-21 August 1998, Everett, WA, Amer. Meteor. Soc., pp.622-625.

Scorer, R. S., 1957: Experiments on convection of isolated masses of buoyant fluid. J. Fluid. Mech, Vol.2, pp.583-596. 